\title{
The field of Nash functions and factorization of polynomials
}

\author{
by StanisŁaw Spodzieja (Łódź)
}

\begin{abstract}
The algebraically closed field of Nash functions is introduced. It is shown that this field is an algebraic closure of the field of rational functions in several variables. We give conditions for the irreducibility of polynomials with Nash coefficients, a description of factors of a polynomial over the field of Nash functions and a theorem on continuity of factors.
\end{abstract}

Introduction. A holomorphic function $f$ in an open connected set $\Omega \subset$ $\mathbb{C}^{m}$ is called a Nash function if there exists an irreducible polynomial $P$ : $\mathbb{C}^{m} \times \mathbb{C} \rightarrow \mathbb{C}$ such that $P(\lambda, f(\lambda))=0$ for $\lambda \in \Omega$. In Section 2 of the paper, the field of Nash functions is introduced (Prop. 2.1). It is an algebraic closure of the field of rational functions (Thm. 2.4 and Cor. 2.5). In the literature it is shown that such a closure is embedded in the field of Puiseux power series ([W], Thm. 3.1, Ch. IV). In the definition of the Nash field, the main trouble is to construct a family of sets $\left\{\Omega_{P}\right\}$ with appropriate properties (Thm. 1.1). The main problem is to obtain the simple connectedness of these sets, which is the key fact in the proof of the algebraic closedness of the Nash field.

In Section 3, conditions of the irreducibility of polynomials with coefficients in the Nash field are given (Thm. 3.2) and, as a corollary, a generalization of the Krull Theorem in the complex domain (Cor. 3.3).

In Section 5, a theorem on the continuity of factors of a decomposition of a polynomial as a function of parameters is given (Thm. 5.1). In the proof of this theorem, the key role is played by an effective interpretation of the well-known fact that an irreducible polynomial which is reducible over the algebraic closure of the field of its coefficients is a product of conjugate polynomials (Thm. 4.4). Here, systems of coefficients of conjugate polynomials form a cycle of Nash mappings.

1991 Mathematics Subject Classification: Primary 12D99; Secondary 12 F99.

Key words and phrases: Nash function, field, decomposition of polynomial.

This research was realized within the project No. 2 P03A 05010 financed in 1996-1998 by KBN. 
For $x=\left(x_{1}, \ldots, x_{n}\right) \in \mathbb{C}^{n}$, we put $|x|=\max _{i=1, \ldots, n}\left|x_{i}\right|$ and $x^{\prime}=$ $\left(x_{1}, \ldots, x_{n-1}\right)$. If $V \subset \mathbb{C}^{n}$, then by $\bar{V}$ we denote the closure of $V$ in the natural topology of $\mathbb{C}^{n}$, and by $\bar{V}^{Z}$ the closure of $V$ in the Zariski topology of $\mathbb{C}^{n}$.

By $\mathrm{K}\left[\Lambda_{1}, \ldots, \Lambda_{m}\right], \mathrm{K}\left(\Lambda_{1}, \ldots, \Lambda_{m}\right), \mathrm{K}\left[\Lambda_{1}, \ldots, \Lambda_{m}\right]^{*}$ we denote the ring of polynomials in variables $\Lambda=\left(\Lambda_{1}, \ldots, \Lambda_{m}\right)$ with coefficients in $\mathrm{K}$, the residue field of this ring, and the set of its nonzero polynomials, respectively. We also write $\mathrm{K}[\Lambda], \mathrm{K}(\Lambda)$ and $\mathrm{K}[\Lambda]^{*}$, respectively.

1. The sets $\Omega_{P}$. In this section we shall define a family of subsets of $\mathbb{C}^{m}$, which will be the domains of Nash functions needed for construction of the Nash field.

1.1. TheOREM. There exists a mapping which assigns to each polynomial $P \in \mathbb{C}\left[\Lambda_{1}, \ldots, \Lambda_{m}\right]^{*}$ a set $\Omega_{P} \subset \mathbb{C}^{m}$ in such a manner that the following conditions are satisfied:

$C_{0} . \quad \Omega_{P} \subset\left\{\lambda \in \mathbb{C}^{m}: P(\lambda) \neq 0\right\}$,

$C_{1} . \quad \Omega_{P} \cap \Omega_{Q}=\Omega_{P Q}$,

$C_{2} . \quad \Omega_{P}$ is a dense subset of $\mathbb{C}^{m}$,

$C_{3} . \quad \Omega_{P}$ is an open, connected and simply connected set.

Moreover, one can require that the above mapping satisfy the additional condition

$C_{4} . \quad \Omega_{P}=\mathbb{C}^{m}$ for $P=$ const.

Before the proof we shall make an auxiliary construction and define the sets $\Omega_{P}$. Let us associate first with each polynomial $P \in \mathbb{C}\left[\Lambda_{1}, \ldots, \Lambda_{m}\right]^{*}$ a set $\Gamma_{P} \subset \mathbb{C}^{m}$ defined by the formula

$$
\Gamma_{P}:=\left\{\left(\lambda_{1}, \ldots, \lambda_{m}\right) \in \mathbb{C}^{m}: P\left(\lambda_{1}, \ldots, \lambda_{m-1}, \lambda_{m}+\gamma\right)=0\right.
$$

By the above definition we immediately obtain

1.2. Lemma. Let $P, Q \in \mathbb{C}\left[\Lambda_{1}, \ldots, \Lambda_{m}\right]^{*}$. Then

(i) $\Gamma_{P Q}=\Gamma_{P} \cup \Gamma_{Q}$,

(ii) if $\left(\lambda_{1}, \ldots, \lambda_{m}\right) \in \mathbb{C}^{m} \backslash \Gamma_{P}$, then, for each $\gamma \in[0, \infty)$, we have $\left(\lambda_{1}, \ldots, \lambda_{m-1}, \lambda_{m}+\gamma\right) \in \mathbb{C}^{m} \backslash \Gamma_{P}$

(iii) the set $\mathbb{C}^{m} \backslash \Gamma_{P}$ is dense in $\mathbb{C}^{m}$.

Let $m>1, P \in \mathbb{C}\left[\Lambda_{1}, \ldots, \Lambda_{m}\right]^{*}$, and

$$
P=P_{0} \Lambda_{m}^{d}+P_{1} \Lambda_{m}^{d-1}+\ldots+P_{d}
$$

where $P_{i} \in \mathbb{C}\left[\Lambda_{1}, \ldots, \Lambda_{m-1}\right]$ for $i=0, \ldots, d, P_{0} \neq 0$. We define $\omega(P):=P_{0}$. If additionally $d=\operatorname{deg}_{\Lambda_{m}} P>0$, we define in $\mathbb{C}^{m-1} \backslash V(\omega(P))$, where 
$V(\omega(P))$ is the set of zeros of $\omega(P)$, a continuous function $\alpha_{P}$ by

$$
\alpha_{P}\left(\lambda^{\prime}\right):=2 \max _{j=1, \ldots, d}\left|\frac{P_{j}\left(\lambda^{\prime}\right)}{P_{0}\left(\lambda^{\prime}\right)}\right|^{1 / j}, \quad \lambda^{\prime} \in \mathbb{C}^{m-1} \backslash V(\omega(P)) .
$$

Let us now define inductively the sets $\Omega_{P} \subset \mathbb{C}^{m}$ for any $P \in$ $\mathbb{C}\left[\Lambda_{1}, \ldots, \Lambda_{m}\right]^{*}$ by

$$
\begin{aligned}
& \Omega_{P}:=\mathbb{C} \backslash \Gamma_{P} \subset \mathbb{C}^{1} \quad \text { for } m=1, \\
& \Omega_{P}:=\left(\mathbb{C}^{m} \backslash \Gamma_{P}\right) \cap\left(\Omega_{\omega(P)} \times \mathbb{C}\right) \subset \mathbb{C}^{m} \quad \text { for } m>1 .
\end{aligned}
$$

1.4. Lemma. If $m>1$ and $P \in \mathbb{C}\left[\Lambda_{1}, \ldots, \Lambda_{m}\right]^{*}, \operatorname{deg}_{\Lambda_{m}} P>0$, then

$$
\Omega_{P} \supset\left\{\left(\lambda^{\prime}, \lambda_{m}\right) \in \mathbb{C}^{m}: \lambda^{\prime} \in \Omega_{\omega(P)} \text { and } \operatorname{Re} \lambda_{m}>\alpha_{P}\left(\lambda^{\prime}\right)\right\} .
$$

P r o o f. Indeed, if $\left(\lambda^{\prime}, \lambda_{m}\right) \notin \Omega_{P}$, then either $P\left(\lambda^{\prime}, \lambda_{m}+\gamma\right)=0$ for some $\gamma \geq 0$, so $\left|\lambda_{m}+\gamma\right| \leq \alpha_{P}\left(\lambda^{\prime}\right)$, i.e. $\operatorname{Re} \lambda_{m} \leq \alpha_{P}\left(\lambda^{\prime}\right)$ or $\lambda^{\prime} \notin \Omega_{\omega(P)}$.

Proof of Theorem 1.1. We shall show that the mapping $P \mapsto \Omega_{P}$ defined by (1.3) satisfies $C_{0}-C_{4}$. From the definition of $\Omega_{P}$ we see that $C_{0}$ and $C_{4}$ are satisfied. We shall prove the remaining conditions by induction with respect to the number of variables $m$. For $m=1$ they are obvious. Let us take any $m \geq 2$. Take arbitrary polynomials $P, Q \in \mathbb{C}\left[\Lambda_{1}, \ldots, \Lambda_{m}\right]^{*}$. Then $\omega(P) \omega(Q)=\omega(P Q)$. So, by Lemma 1.2(i) and the induction hypothesis, we get

$$
\begin{aligned}
\Omega_{P Q} & =\left(\mathbb{C}^{m} \backslash\left(\Gamma_{P} \cup \Gamma_{Q}\right)\right) \cap\left(\Omega_{\omega(P) \omega(Q)} \times \mathbb{C}\right) \\
& =\left(\mathbb{C}^{m} \backslash \Gamma_{P}\right) \cap\left(\mathbb{C}^{m} \backslash \Gamma_{Q}\right) \cap\left(\Omega_{\omega(P)} \times \mathbb{C}\right) \cap\left(\Omega_{\omega(Q)} \times \mathbb{C}\right)=\Omega_{P} \cap \Omega_{Q},
\end{aligned}
$$

which gives $C_{1}$.

If $\operatorname{deg}_{\Lambda_{m}} P=0$, then, obviously, $\Omega_{P}=\Omega_{\omega(P)} \times \mathbb{C}$, so, by the induction hypothesis, we see that $C_{2}, C_{3}$ are satisfied. Thus, consider the case $\operatorname{deg}_{\Lambda_{m}} P>0$.

By the induction hypothesis, we see that $\Omega_{\omega(P)}$ is an open and dense subset of $\mathbb{C}^{m-1}$. Since, by Lemma 1.2 (iii), $\mathbb{C}^{m} \backslash \Gamma_{P}$ is dense in $\mathbb{C}^{m}$, therefore $\Omega_{P}$ is dense in $\mathbb{C}^{m}$. This gives $C_{2}$.

We now prove $C_{3}$. First, we show that $\Omega_{P}$ is open. Note that the following inclusion holds:

$$
\bar{\Gamma}_{P} \subset \Gamma_{P} \cup(V(\omega(P)) \times \mathbb{C}) .
$$

Indeed, take any sequence $\lambda^{n} \in \Gamma_{P}$ convergent to $\lambda^{0}=\left(\lambda^{0 \prime}, \lambda_{m}^{0}\right) \in \mathbb{C}^{m}$. Then $\lambda^{n}=\left(\lambda^{n \prime}, \lambda_{m}^{n}-\gamma^{n}\right)$ where $P\left(\lambda^{n \prime}, \lambda_{m}^{n}\right)=0, \gamma^{n} \in[0, \infty)$. If $\lambda_{m}^{n}$ is bounded, then taking a subsequence if necessary, we may assume that $\lambda_{m}^{n} \rightarrow$ $\widetilde{\lambda}_{m}$ where $\widetilde{\lambda}_{m} \in \mathbb{C}$. So, there exists $\widetilde{\gamma} \in[0, \infty)$ such that $\gamma^{n} \rightarrow \widetilde{\gamma}$. Hence $P\left(\lambda^{0 \prime}, \widetilde{\lambda}_{m}\right)=0$, i.e. $P\left(\lambda^{0 \prime}, \lambda_{m}^{0}+\widetilde{\gamma}\right)=0$. This gives $\lambda^{0} \in \Gamma_{P}$. If $\lambda_{m}^{n}$ is not bounded, then taking a subsequence if necessary, we may assume that $\lambda_{m}^{n} \rightarrow \infty$. Hence $\alpha_{P}\left(\lambda^{n \prime}\right) \rightarrow \infty$, therefore $\omega(P)\left(\lambda^{n \prime}\right) \rightarrow 0$. In consequence, 
$\lambda^{0 \prime} \in V(\omega(P))$. Summing up, we have obtained the desired inclusion. Since $V(\omega(P))$ is disjoint from $\Omega_{\omega(P)}$, therefore, by the above, we obtain

$$
\Omega_{P}=\left(\mathbb{C}^{m} \backslash \bar{\Gamma}_{P}\right) \cap\left(\Omega_{\omega(P)} \times \mathbb{C}\right) .
$$

Hence, by the induction hypothesis, we have the openness of $\Omega_{P}$.

We now prove the connectedness of $\Omega_{P}$. Take any $\lambda^{0}=\left(\lambda^{0 \prime}, \lambda_{m}^{0}\right), \lambda^{1}=$ $\left(\lambda^{1 \prime}, \lambda_{m}^{1}\right) \in \Omega_{P}$. We show that these points may be joined by a curve lying in $\Omega_{P}$. Let $r:=1+\max \left(\alpha_{P}\left(\lambda^{0 \prime}\right), \alpha_{P}\left(\lambda^{1 \prime}\right), \operatorname{Re} \lambda_{m}^{0}, \operatorname{Re} \lambda_{m}^{1}\right)$. By Lemmas 1.2(ii) and 1.4, without loss of generality, we may assume that $\lambda_{m}^{0}=\lambda_{m}^{1}=r$. Since $\lambda^{0 \prime}, \lambda^{1 \prime} \in \Omega_{\omega(P)}$, by the induction hypothesis, there exists a curve $\tau:[\alpha, \beta] \rightarrow \Omega_{\omega(P)}$ joining $\lambda^{0 \prime}$ and $\lambda^{1 \prime}$. Take the curve

$$
\sigma(\xi):=\left(\tau(\xi), \max \left(r, \alpha_{P}(\tau(\xi))+1 / 2\right)\right), \quad \xi \in[\alpha, \beta] .
$$

Since $\max \left(r, \alpha_{P}(\tau(\xi))+1 / 2\right)>\alpha_{P}(\tau(\xi))$ for $\xi \in[\alpha, \beta]$, therefore, by Lemma 1.4, we see that $\sigma$ lies in $\Omega_{P}$ and, obviously, joins $\lambda^{0}$ and $\lambda^{1}$. Summing up, $\Omega_{P}$ is a connected set.

We now prove the simple connectedness of $\Omega_{P}$. Take any loop $\sigma=$ $\left(\sigma^{\prime}, \sigma_{m}\right):[\alpha, \beta] \rightarrow \Omega_{P}$ where $\sigma^{\prime}:[\alpha, \beta] \rightarrow \mathbb{C}^{m-1}, \sigma_{m}:[\alpha, \beta] \rightarrow \mathbb{C}$. Of course, $\sigma^{\prime}$ is a loop lying in $\Omega_{\omega(P)}$. Let $r:=1+\sup \left(\left\{\alpha_{P}\left(\sigma^{\prime}(\xi)\right): \xi \in\right.\right.$ $\left.[\alpha, \beta]\} \cup\left\{\operatorname{Re} \sigma_{m}(\xi): \xi \in[\alpha, \beta]\right\}\right)$. By Lemmas 1.2(ii) and 1.4, without loss of generality, we may assume that $\sigma_{m}(\xi)=r$ for $\xi \in[\alpha, \beta]$. By the induction hypothesis, we see that there exists a homotopy $H^{\prime}:[\alpha, \beta] \times[0,1] \rightarrow \Omega_{\omega(P)}$ of $\sigma^{\prime}$ to a point. Take the mapping

$H(\xi, \gamma):=\left(H^{\prime}(\xi, \gamma), \max \left(r, \alpha_{P}\left(H^{\prime}(\xi, \gamma)\right)+1 / 2\right)\right), \quad(\xi, \gamma) \in[\alpha, \beta] \times[0,1]$.

By Lemma 1.4, we see that $H$ is a homotopy of $\sigma$ to a constant curve $[\alpha, \beta] \ni \xi \mapsto H(\xi, 1)$ in $\Omega_{P}$. This gives $C_{3}$.

The proof of Theorem 1.1 is complete.

2. The field of Nash functions. In this section we define the algebraically closed field of Nash functions. The basic properties of Nash functions can be found, for example, in [T].

In the sequel, let $m$ be a fixed positive integer, and $\Lambda$ a system of $m$ variables $\Lambda_{1}, \ldots, \Lambda_{m}$. Let

$$
\mathbb{C}[\Lambda]^{*} \ni P \mapsto \Omega_{P} \subset \mathbb{C}^{m}
$$

be a mapping satisfying conditions $C_{0}-C_{3}$ of Theorem 1.1.

Let $P \in \mathbb{C}[\Lambda]^{*}$. From conditions $C_{2}$ and $C_{3}$ we see that $\Omega_{P} \neq \emptyset$ is a connected open set. Consequently, the set $\mathcal{N}_{P}$ of all Nash functions in $\Omega_{P}$ is a ring with the usual operations of addition and multiplication (see [T], Cor. 1.11 or $[\mathrm{Sp}]$, Thm. 3.3) and, moreover, it is a domain. In the set

$$
\bigcup_{P \in \mathbb{C}[\Lambda]^{*}} \mathcal{N}_{P}
$$


we introduce a relation " $\sim$ " in the following way:

$$
\begin{aligned}
\left(f_{1}: \Omega_{P} \rightarrow\right. & \mathbb{C}) \sim\left(f_{2}: \Omega_{Q} \rightarrow \mathbb{C}\right) \\
& \Leftrightarrow \text { there exists } \Omega_{R} \subset \Omega_{P} \cap \Omega_{Q} \text { such that }\left.f_{1}\right|_{\Omega_{R}}=\left.f_{2}\right|_{\Omega_{R}} .
\end{aligned}
$$

From conditions $C_{0}-C_{3}$ we immediately see that " $\sim$ " is an equivalence relation. The equivalence class determined by $f: \Omega_{P} \rightarrow \mathbb{C}$ is denoted by $[f]$ and the set of all such classes by $\mathcal{N}$.

2.1. Proposition. The set $\mathcal{N}$ together with the operations "+", "." defined by

$$
\left[f_{1}\right]+\left[f_{2}\right]:=\left[\left.f_{1}\right|_{\Omega_{P Q}}+\left.f_{2}\right|_{\Omega_{P Q}}\right], \quad\left[f_{1}\right] \cdot\left[f_{2}\right]:=\left[\left.\left.f_{1}\right|_{\Omega_{P Q}} f_{2}\right|_{\Omega_{P Q}}\right],
$$

where $f_{1} \in \mathcal{N}_{P}, f_{2} \in \mathcal{N}_{Q}$, is a field.

Moreover, the field $\mathbb{C}(\Lambda)$ of rational functions is embedded in $\mathcal{N}$ by

$$
\mathbb{C}(\Lambda) \ni \frac{P}{Q} \mapsto\left[\left.\frac{P}{Q}\right|_{\Omega_{Q}}\right] \in \mathcal{N} .
$$

The field $\mathcal{N}$ is called the field of Nash functions or, briefly, Nash field.

The proof of the proposition will be preceded by

2.2. LEMmA. If $f \in \mathcal{N}_{P}, f \neq 0$, then there exists $Q \in \mathbb{C}[\Lambda]^{*}$ such that the set of zeros of $f$ is contained in the set of zeros of $Q$, and $1 /\left.f\right|_{\Omega_{P Q}} \in \mathcal{N}_{P Q}$.

Proof. Let $R \in \mathbb{C}[\Lambda, Z]$, where $Z$ denotes a single variable, be an irreducible polynomial such that $R(\lambda, f(\lambda))=0$ for $\lambda \in \Omega_{P}$. Hence we conclude that the set of zeros of $f$ is contained in the set of zeros of $Q:=R(\Lambda, 0) \in \mathbb{C}[\Lambda]$ and, obviously, $Q \neq 0$. So, by condition $C_{0}$, we have $f(\lambda) \neq 0$ for $\lambda \in \Omega_{P Q}$. This and Thm. 1.10 in [T] or Cor. 4.10 in [Sp] give $1 /\left.f\right|_{\Omega_{P Q}} \in \mathcal{N}_{P Q}$, which ends the proof.

Proof of Proposition 2.1. Since $\mathcal{N}_{P Q}$ is a domain, therefore, by conditions $C_{0}-C_{3}$, we see that the operations "+" and "." are well defined and that $\mathcal{N}$ is a domain. By Lemma 2.2 any nonzero element $[f] \in \mathcal{N}$ is invertible. This gives the first part of the proposition. The second part is obvious.

2.3. Remark. In the above proposition we have used all conditions $C_{0}-C_{3}$ except the simple connectedness of $\Omega_{P}$. The last property is the key fact in the proof that $\mathcal{N}$ is algebraically closed. Omitting the simple connectedness, we could take instead of $\left\{\Omega_{P}\right\}$, for example, the family of sets $\left\{\lambda \in \mathbb{C}^{m}: P(\lambda) \neq 0\right\}, P \in \mathbb{C}[\Lambda]^{*}$. But this would lead us only to the field of rational functions.

2.4. THEOREM. The field $\mathcal{N}$ is algebraically closed. 
Proof. Take an irreducible polynomial $\mathfrak{p} \in \mathcal{N}[Z]$ in one variable $Z$. Then

$$
\mathfrak{p}(Z)=\left[a_{0}\right] Z^{d}+\left[a_{1}\right] Z^{d-1}+\ldots+\left[a_{d}\right], \quad d \geq 1,\left[a_{0}\right] \neq[0] .
$$

By condition $C_{1}$, there exists $P$ such that $a_{j} \in \mathcal{N}_{P}$ for $j=0, \ldots, d$. Take the pseudopolynomial

$$
g(\lambda, Z):=a_{0}(\lambda) Z^{d}+a_{1}(\lambda) Z^{d-1}+\ldots+a_{d}(\lambda), \quad \lambda \in \Omega_{P} .
$$

Since $\mathfrak{p}$ is irreducible, its discriminant is nonzero in $\mathcal{N}$. So, by Lemma 2.2 and conditions $C_{0}$ and $C_{1}$, we may assume that the discriminant of $g$ does not vanish in $\Omega_{P}$. In consequence, by the implicit function theorem, the projection $\pi: W \ni(\lambda, z) \mapsto \lambda \in \Omega_{P}$, where $W=\left\{(\lambda, z) \in \Omega_{P} \times \mathbb{C}\right.$ : $g(\lambda, z)=0\}$, is a covering. Since $\Omega_{P}$ is simply connected (by condition $C_{3}$ ), so, by the monodromy theorem ([N], Cor., p. 20), there exists a holomorphic function $f: \Omega_{P} \rightarrow \mathbb{C}$ such that $g(\lambda, f(\lambda))=0$ for $\lambda \in \Omega_{P}$. Moreover, by Prop. 1.6 in $[\mathrm{T}], f$ is a Nash function. So, we obtain $\mathfrak{p}([f])=[0]$. Therefore, $\mathcal{N}$ is an algebraically closed field.

Since each element of the field $\mathcal{N}$ is algebraic over $\mathbb{C}(\Lambda)$, by the above theorem and Proposition 2.1 we easily obtain

2.5. Corollary. $\mathcal{N}$ is the algebraic closure of the field $\mathbb{C}(\Lambda)$ of rational functions.

3. Irreducibility of polynomials with Nash coefficients. In the sequel, let $n$ be a fixed positive integer and $X$ a system of $n$ variables $X_{1}, \ldots, X_{n}$.

By $I$ we denote a multiindex $\left(i_{1}, \ldots, i_{n}\right)$ where $i_{j} \in \mathbb{Z}, i_{j} \geq 0$, and $\|I\|=i_{1}+\ldots+i_{n} . X^{I}$ denotes the monomial $X_{1}^{i_{1}} \ldots X_{n}^{i_{n}}$.

By $Y$ we denote the system of variables $Y=\left(Y_{I}:\|I\| \leq d\right)$ where $d$ is a positive integer. The number of these variables is equal to $\left(\begin{array}{c}d+n \\ n\end{array}\right)$ and it is the number of $n$-variable monomials of degree not exceeding $d$. By $\mathcal{G}_{d}$ we denote the polynomial from $\mathbb{Z}[Y, X]$ of the form

$$
\mathcal{G}_{d}(Y, X):=\sum_{\|I\| \leq d} Y_{I} X^{I} .
$$

Let $Q \in \mathbb{C}[\Lambda]^{*}$ and $g \in \mathcal{N}_{Q}[X]$. The set of those $\lambda \in \Omega_{Q}$ for which the polynomial $g(\lambda, X)$ is reducible in $\mathbb{C}[X]$ is called the spectrum of $g$ and denoted by $\operatorname{Spec} g$ (cf. $[\mathrm{St}])$.

3.1. Proposition. Let $g \in \mathcal{N}_{Q}[X]$. Then either Spec $g$ is contained in a proper algebraic subset of $\mathbb{C}^{m}$, or is a dense subset of $\Omega_{Q}$ and then

$$
\left\{\lambda \in \Omega_{Q}: \operatorname{deg} g(\lambda, X)=\operatorname{deg} g\right\} \subset \operatorname{Spec} g .
$$


Proof. Let $d:=\operatorname{deg} g$ and $g=\mathcal{G}_{d}(F, X)$ where $F=\left(f_{I}:\|I\| \leq d\right)$ : $\Omega_{Q} \rightarrow \mathbb{C}\left(\begin{array}{c}d+n \\ n\end{array}\right)$ is a Nash mapping. Then, for any $H \in \mathbb{Z}[Y]$, we have $H \circ F \in$ $\mathcal{N}_{Q}$. So, by Lemma 2.2, the set of zeros of the function $H \circ F$ is identical with $\Omega_{Q}$ or is contained in some proper algebraic subset of $\mathbb{C}^{m}$. Moreover, the set $\left\{\lambda \in \Omega_{Q}: \operatorname{deg} g(\lambda, X)<\operatorname{deg} g\right\}$ is equal to $\left\{\lambda \in \Omega_{Q}: f_{I}(\lambda)=0,\|I\|=d\right\}$. So, by Lemma 2.2 , it is contained in some proper algebraic subset of $\mathbb{C}^{m}$. In consequence, applying the Noether Theorem ([Sc], Sec. 10, Thm. 15), we easily obtain the assertion.

If $F=\left(f_{I}:\|I\| \leq d\right): \Omega_{Q} \rightarrow \mathbb{C}^{\left(\begin{array}{c}d+n \\ n\end{array}\right)}$ is a Nash mapping, then we put $[F]:=\left(\left[f_{I}\right]:\|I\| \leq d\right)$.

3.2. TheOREM. Let $F: \Omega_{Q} \rightarrow \mathbb{C}^{\left(\begin{array}{c}d+n \\ n\end{array}\right)}$, where $d \in \mathbb{N}$, be a Nash mapping and let $g \in \mathcal{N}_{Q}[X]$ and $\mathfrak{g} \in \mathcal{N}[X]$ be the polynomials $g(X):=\mathcal{G}_{d}(F, X)$, $\mathfrak{g}:=\mathcal{G}_{d}([F], X)$. Then the following conditions are equivalent:

(i) $\mathfrak{g}$ is irreducible in $\mathcal{N}[X]$,

(ii) there exists $R \in \mathbb{C}[\Lambda]^{*}$ such that $\Omega_{R} \subset \Omega_{Q}$ and, for each $\lambda \in \Omega_{R}$, the polynomial $g(\lambda, X)$ is irreducible in $\mathbb{C}[X]$,

(iii) there exists $\lambda \in \Omega_{Q}$ such that $\operatorname{deg} g(\lambda, X)=\operatorname{deg} g$ and the polynomial $g(\lambda, X)$ is irreducible in $\mathbb{C}[X]$.

Proof. (i) $\Rightarrow$ (ii). Let $H \in \mathbb{Z}[Y]$. Then $H \circ F \in \mathcal{N}_{Q}$. So, if $H \circ F \neq 0$, then, by Lemma 2.2 , there exists $R \in \mathbb{C}[\Lambda]^{*}$ such that $\Omega_{R} \subset \Omega_{Q}$ and $H \circ F(\lambda) \neq 0$ for $\lambda \in \Omega_{R}$. This, together with Thm. 2.4 and the Noether Theorem, gives (ii).

(ii) $\Rightarrow$ (iii). Obvious.

(iii) $\Rightarrow$ (i). Otherwise, the set Spec $g$ would be a dense subset of $\Omega_{Q}$, which would contradict Proposition 3.1.

Let us take $Q=1$. Then we may assume that $\Omega_{Q}=\mathbb{C}^{m}$ (Thm. 1.1, $\left.C_{4}\right)$. Hence for $P \in \mathbb{C}[\Lambda, X] \subset \mathcal{N}_{Q}[X]$ we have Spec $P=\left\{\lambda \in \mathbb{C}^{m}: P(\lambda, X)\right.$ is reducible in $\mathbb{C}[X]\}$. Hence, from the above theorem and Proposition 3.1, we immediately obtain the Krull Theorem in the complex domain (see $[\mathrm{K}]$ and [Sc], Sec. 10, Cor. 2).

3.3. Corollary. Let $P \in \mathbb{C}[\Lambda, X]$ be a polynomial of positive degree with respect to $X$. Then the following conditions are equivalent:

(i) $P$ is irreducible in $\mathcal{N}[X]$,

(ii) Spec $P$ is contained in some proper algebraic subset of $\mathbb{C}^{m}$,

(iii) there exists $\lambda \in \mathbb{C}^{m}$ such that $\operatorname{deg}_{X} P(\lambda, X)=\operatorname{deg}_{X} P$ and the polynomial $P(\lambda, X)$ is irreducible in $\mathbb{C}[X]$.

4. Cycle of Nash mappings. Let $Q \in \mathbb{C}[\Lambda]^{*}, d \in \mathbb{N}$. We say that the system of Nash mappings $F_{i}=\left(f_{i, I}:\|I\| \leq d\right): \Omega_{Q} \rightarrow \mathbb{C}^{\left(\begin{array}{c}d+n \\ n\end{array}\right)}, i=1, \ldots, k$, 
is a cycle if

$$
P(X, Z):=\prod_{i=1}^{k}\left(Z-\mathcal{G}_{d}\left(\left[F_{i}\right], X\right)\right)
$$

is an irreducible polynomial in $\mathbb{C}(\Lambda)[X, Z]$ (i.e. $\mathcal{G}_{d}\left(\left[F_{i}\right], X\right), i=2, \ldots, k$, are all the conjugates of $\mathcal{G}_{d}\left(\left[F_{1}\right], X\right)$ in $\mathcal{N}[X]$ over $\left.\mathbb{C}(\Lambda)\right)$.

Conjugate polynomials over $\mathbb{C}(\Lambda)$ have the following geometrical interpretation.

4.1. Proposition. Let $F_{i}=\left(f_{i, I}:\|I\| \leq d\right): \Omega_{Q} \rightarrow \mathbb{C}^{\left(\begin{array}{c}d+n \\ n\end{array}\right)}, i=1,2$, be Nash mappings. Then the polynomials $\mathcal{G}_{d}\left(\left[F_{i}\right], X\right), i=1,2$, in $\mathcal{N}[X]$ are

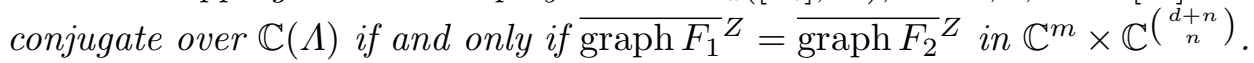

Proof. Let $\mathcal{G}_{d}\left(\left[F_{i}\right], X\right), i=1,2$, be conjugate over $\mathbb{C}(\Lambda)$. Then, there exists an automorphism $\varphi: \mathcal{N} \rightarrow \mathcal{N}$ such that $\left.\varphi\right|_{\mathbb{C}(\Lambda)}: \mathbb{C}(\Lambda) \rightarrow \mathbb{C}(\Lambda)$ is the identity and $\left[F_{2}\right]=\left(\varphi\left(\left[f_{1, I}\right]\right):\|I\| \leq d\right)$. Let $V_{i}:=\overline{\operatorname{graph} F_{i}}{ }^{Z}$, and $I_{i}$ be the ideal of $V_{i}, i=1,2$. Then, for any polynomial $P \in I_{1}, P\left(\Lambda,\left[F_{2}\right]\right)=$ $P\left(\Lambda,\left(\varphi\left(\left[f_{1, I}\right]\right):\|I\| \leq d\right)\right)=0$. This gives that $V_{2} \subset V_{1}$. Hence, by symmetry of conditions we have $V_{1}=V_{2}$.

Assume now that $\frac{1}{\text { graph } F_{1}} Z=\overline{\text { graph } F_{2}} Z$. By [T], Prop. 1.6, it is an irreducible algebraic set. Let $p_{i}(X):=\mathcal{G}_{d}\left(F_{i}, X\right) \in \mathcal{N}_{Q}[X], i=1,2$. Then we easily see that $\overline{\operatorname{graph} p_{1}} Z=\overline{\operatorname{graph} p_{2}} Z$ is an irreducible algebraic set in $\mathbb{C}^{m} \times \mathbb{C}^{n} \times \mathbb{C}$. So, there exists an irreducible polynomial $P \in \mathbb{C}[\Lambda, X, Z]$ such that $P\left(\lambda, x, p_{i}(\lambda, x)\right)=0$ for $(\lambda, x) \in \Omega_{Q} \times \mathbb{C}^{n}, i=1,2$. This implies that $\mathcal{G}_{d}\left(\left[F_{i}\right], X\right), i=1,2$, are conjugate over $\mathbb{C}(\Lambda)$.

4.2. Proposition. If $F_{i}: \Omega_{Q} \rightarrow \mathbb{C}^{\left(\begin{array}{c}d+n \\ n\end{array}\right)}, i=1, \ldots, k$ is a cycle, then $\bigcup_{i=1}^{k}$ graph $F_{i}$ is a dense subset of the $m$-dimensional irreducible algebraic set $W:=\overline{\operatorname{graph} F_{1}} Z$.

Proof. Let $\pi: W \ni(\lambda, y) \mapsto \lambda \in \mathbb{C}^{m}$ be the canonical projection. By the definition of $W$, we easily see that it is an irreducible algebraic set, $\operatorname{dim} W=$ $m$ (see $[\mathrm{T}]$, Prop. 1.6) and $\pi$ is a dominating mapping. Consequently, we may assume that $\left.\pi\right|_{U}: U \rightarrow \Omega_{Q}$, where $U:=\pi^{-1}\left(\Omega_{Q}\right)$, is a covering (by conditions $C_{0}, C_{1}$, [M], Cor. 3.17 and Fundamental Openness Principle 3.10). Moreover, by the density of the set $\Omega_{Q}$ in $\mathbb{C}^{m}$ (condition $C_{2}$ ), we see that $U$ is dense in $W$ (by using additionally e.g. [M], Thm. 2.33).

It suffices to show that

$$
U=\bigcup_{i=1}^{k} \operatorname{graph} F_{i}
$$

Indeed, by the definition of a cycle and Proposition 4.1, we have $\bigcup_{i=1}^{k} \operatorname{graph} F_{i}$ $\subset U$. Suppose that the equality in (4.3) does not hold. Then, from the above, 
using the facts that $\Omega_{Q}$ is an open, connected and simply connected set (condition $C_{3}$ ) and $\left.\pi\right|_{U}$ is a covering, by the monodromy theorem, there exists a holomorphic mapping $F: \Omega_{Q} \rightarrow \mathbb{C}^{\left(\begin{array}{c}d+n \\ n\end{array}\right)}$, different from $F_{i}, i=1, \ldots, k$, such that graph $F \subset W$. Thus, by [T], Prop. 1.6, $F$ is a Nash mapping. Moreover, by Proposition $4.1, \mathcal{G}_{d}([F], X)$ is a conjugate to $\mathcal{G}_{d}\left(\left[F_{1}\right], X\right)$ over $\mathbb{C}(\Lambda)$, and different from all $\mathcal{G}_{d}\left(\left[F_{i}\right], X\right), i=1, \ldots, k$. This contradicts the definition of a cycle. Summing up, we have (4.3).

4.4. Theorem. If $P \in \mathbb{C}[\Lambda, X]$ is an irreducible polynomial in $\mathbb{C}[\Lambda, X]$, monic with respect to $X_{1}$, whose spectrum is a dense subset of $\mathbb{C}^{m}$, then $P$ is reducible in $\mathcal{N}[X]$ and there exists a cycle $F_{i}=\left(f_{i, I}:\|I\| \leq d\right): \Omega_{Q} \rightarrow$

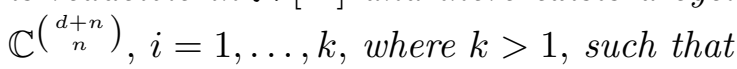

$$
P(\lambda, X)=\prod_{i=1}^{k} \mathcal{G}_{d}\left(F_{i}(\lambda), X\right) \quad \text { for } \lambda \in \Omega_{Q}
$$

is a decomposition of $P(\lambda, X)$ into distinct irreducible factors in $\mathbb{C}[X]$, monic with respect to $X_{1}$. In particular,

$$
P(X)=\prod_{i=1}^{k} \mathcal{G}_{d}\left(\left[F_{i}\right], X\right)
$$

is a decomposition of $P$ into distinct irreducible factors in $\mathcal{N}[X]$.

Proof. The reducibility of $P$ in $\mathcal{N}[X]$ follows from Corollary 3.3. Let $\mathfrak{p}_{1}$ be an irreducible factor of $P$ in $\mathcal{N}[X]$ monic with respect to $X_{1}$. Let $\mathfrak{p}_{2}, \ldots, \mathfrak{p}_{k}$ be all the conjugates of $\mathfrak{p}_{1}$ in $\mathcal{N}[X]$ over $\mathbb{C}(\Lambda)$. Then $\mathfrak{p}_{i}$ are irreducible, monic with respect to $X_{1}$ and $\mathfrak{p}_{1} \ldots \mathfrak{p}_{k} \in \mathbb{C}(\Lambda)[X]$. Moreover, $\mathfrak{p}_{i}$ are divisors of $P$ in $\mathcal{N}[X]$. In consequence, from irreducibility of $P$ in $\mathbb{C}[\Lambda, X]$, we have $P=\mathfrak{p}_{1} \ldots \mathfrak{p}_{k}$. Then there exists a cycle $F_{i}: \Omega_{Q} \rightarrow \mathbb{C}^{\left(\begin{array}{c}d+n \\ n\end{array}\right),}$ $i=1, \ldots, k$, such that $\mathfrak{p}_{i}=\mathcal{G}_{d}\left(\left[F_{i}\right], X\right), i=1, \ldots, k$. By conditions $C_{0}, C_{1}$ and Theorem 3.2 , we may assume that $\mathcal{G}_{d}\left(F_{i}(\lambda), X\right), i=1, \ldots, k$, are irreducible in $\mathbb{C}[X]$ for $\lambda \in \Omega_{Q}$. Moreover, by the irreducibility of $P$ in $\mathbb{C}[\Lambda, X]$, one can assume that, for $\lambda \in \Omega_{Q}$, the discriminant of $P(\lambda, X)$ with respect to $X_{1}$ does not vanish, i.e. $\mathcal{G}_{d}\left(F_{i}(\lambda), X\right), i=1, \ldots, k$, are distinct in $\mathbb{C}[X]$ for $\lambda \in \Omega_{Q}$. Hence we easily conclude that $\mathfrak{p}_{i}, i=1, \ldots, k$, are distinct in $\mathcal{N}[X]$. Summing up, we have the assertion of the theorem.

5. Continuous dependence of factors of a decomposition of a polynomial on parameters. We now give a theorem on the continuous dependence of factors of a decomposition of a polynomial on parameters.

The definition of the multiplicity of a mapping and the notation are taken from [M], Def. 3.12. 
5.1. Theorem. If $P \in \mathbb{C}[\Lambda, X]$ is an irreducible polynomial in $\mathbb{C}[\Lambda, X]$ whose spectrum is dense in $\mathbb{C}^{m}$, then there exist a positive integer $d$, an $m$ dimensional irreducible algebraic set $W \subset \mathbb{C}^{m} \times \mathbb{C}^{\left(\begin{array}{c}d+n \\ n\end{array}\right)}$ and a polynomial $G \in \mathbb{C}[\Lambda]^{*}$ such that the canonical projection $\pi: W \ni(\lambda, y) \mapsto \lambda \in \mathbb{C}^{m}$ is proper and

$$
G(\lambda) P(\lambda, X)=\prod_{(\lambda, y) \in \pi^{-1}(\lambda)} \mathcal{G}_{d}^{\text {mult }_{(\lambda, y)}(\pi)}(y, X), \quad \lambda \in \mathbb{C}^{m} .
$$

Moreover, there exists an algebraic set $V \varsubsetneqq \mathbb{C}^{m}$ such that, for $(\lambda, y) \in$ $\pi^{-1}(\lambda), \lambda \in \mathbb{C}^{m} \backslash V$, we have $\operatorname{mult}_{(\lambda, y)}(\pi)=1$ and the right-hand side of $(5.2)$ is a decomposition of $G(\lambda) P(\lambda, X)$ into nonassociated irreducible factors in $\mathbb{C}[X]$.

What is more, if $P$ is monic with respect to one of the variables $X_{i}$, $i=1, \ldots, n$, then one can take $G=1$.

5.3. Remark. The continuity mentioned in the title of the section follows from the fact that $\pi$ is a branched covering.

5.4. Example. The polynomial $P\left(\Lambda_{1}, \Lambda_{2}, X_{1}\right):=\Lambda_{1} X_{1}^{2}-\Lambda_{2}$ is irreducible in $\mathbb{C}\left[\Lambda_{1}, \Lambda_{2}, X_{1}\right]$ and its spectrum is dense in $\mathbb{C}^{2}$. We shall show that in decomposition (5.2) we cannot take $G\left(\Lambda_{1}, \Lambda_{2}\right)=1$. In fact, otherwise, for some $W$ and $V$,

$$
\lambda_{1} X_{1}^{2}-\lambda_{2}=\left(y_{1} X_{1}+y_{2}\right)\left(y_{1}^{*} X_{1}+y_{2}^{*}\right)
$$

for $\left(\lambda_{1}, \lambda_{2}, y_{1}, y_{2}\right),\left(\lambda_{1}, \lambda_{2}, y_{1}^{*}, y_{2}^{*}\right) \in W,\left(\lambda_{1}, \lambda_{2}\right) \notin V$. Then $y_{1}, y_{1}^{*}$ would satisfy the equation $Y^{2}+\alpha\left(\Lambda_{1}, \Lambda_{2}\right) Y+\Lambda_{1}=0$, and $y_{2}, y_{2}^{*}$ would satisfy the equation $Y^{2}+\beta\left(\Lambda_{1}, \Lambda_{2}\right) Y-\Lambda_{2}=0$, for some $\alpha, \beta \in \mathbb{C}\left[\Lambda_{1}, \Lambda_{2}\right]$. Using the Viète formulae, we would easily obtain $4 \Lambda_{1} \Lambda_{2}+\Lambda_{1} \beta^{2}\left(\Lambda_{1}, \Lambda_{2}\right)-$ $\Lambda_{2} \alpha^{2}\left(\Lambda_{1}, \Lambda_{2}\right)=0$, which is impossible.

Taking $G\left(\Lambda_{1}, \Lambda_{2}\right):=\Lambda_{1}, W:=\left\{\left(\lambda_{1}, \lambda_{2}, y_{1}, y_{2}\right) \in \mathbb{C}^{2} \times \mathbb{C}^{2}: y_{1}=\lambda_{1}, y_{2}^{2}=\right.$ $\left.\lambda_{1} \lambda_{2}\right\}, V:=\left\{\left(\lambda_{1}, \lambda_{2}\right) \in \mathbb{C}^{2}: \lambda_{1} \lambda_{2}=0\right\}$ we have decomposition (5.2)

$$
G\left(\lambda_{1}, \lambda_{2}\right) P\left(\lambda_{1}, \lambda_{2}, X_{1}\right)=\left(\lambda_{1} X_{1}+\sqrt{\lambda_{1} \lambda_{2}}\right)\left(\lambda_{1} X_{1}-\sqrt{\lambda_{1} \lambda_{2}}\right) .
$$

The proof of Theorem 5.1 will be preceded by two lemmas.

5.5. Lemma. Let $P \in \mathbb{C}[X]$ be a polynomial monic with respect to $X_{1}$, $d:=\operatorname{deg} P>0$. Then, if the coefficients of $P$ are bounded by $r \geq 1$, then the coefficients of any factor of $P$ monic with respect to $X_{1}$ are bounded by

$$
B_{d, n}(r):=\max _{1 \leq k \leq j \leq d}\left(\begin{array}{l}
j \\
k
\end{array}\right)\left(2(d+1)^{n-1} r\right)^{k} .
$$

Proof. The proof is by induction on the number $n$ of variables. If $n=1$, then the zeros of $P$ are bounded by $2 r$ (since $r \geq 1$ ). Hence from the Viète formulae we have the assertion for $n=1$. Let $P \in \mathbb{C}\left[X, X_{n+1}\right]$ be a polynomial satisfying the assumptions of the lemma, and let $E$ be its factor, monic 
with respect to $X_{1}$. Let

$$
P\left(X, X_{n+1}\right)=\sum_{\|I\| \leq d}\left(\alpha_{0, I} X_{n+1}^{d}+\alpha_{1, I} X_{n+1}^{d-1}+\ldots+\alpha_{d, I}\right) X^{I}
$$

and

$$
E\left(X, X_{n+1}\right)=\sum_{\|I\| \leq d}\left(\beta_{0, I} X_{n+1}^{d}+\beta_{1, I} X_{n+1}^{d-1}+\ldots+\beta_{d, I}\right) X^{I},
$$

where $\alpha_{i, I}, \beta_{i, I} \in \mathbb{C}$ for $\|I\| \leq d, i=1, \ldots, d$. Let $\varepsilon$ be a $(d+1)$ th primitive root of unity. Put $P_{i}(X)=P\left(X, \varepsilon^{i}\right), E_{i}(X)=E\left(X, \varepsilon^{i}\right)$, for $i=1, \ldots, d+1$. Then, by (5.6), we see that the coefficients of $P_{i}$ are bounded by $(d+1) r$, and that $E_{i}$ is a factor of $P_{i}$ monic with respect to $X_{1}, i=1, \ldots, d+1$. So, by the induction hypothesis, the coefficients of $E_{i}$ are bounded by $B_{\operatorname{deg} P_{i}, n}((d+$ $1) r) \leq B_{d, n}((d+1) r)=B_{d, n+1}(r)$. Let $E_{i}(X)=\sum_{\|I\| \leq d} \gamma_{i, I} X^{I}, i=$ $1, \ldots, d+1$, where $\gamma_{i, I} \in \mathbb{C}$ for $\|I\| \leq d, i=1, \ldots, d+1$. Let $L: \mathbb{C}^{d+1} \rightarrow \mathbb{C}^{d+1}$ be a linear mapping represented by the matrix $\mathcal{A}:=\left[\varepsilon^{i(d+1-j)}\right]_{i, j=1, \ldots, d+1}$. By (5.7), we have $L\left(\beta_{0, I}, \ldots, \beta_{d, I}\right)=\left(\gamma_{1, I}, \ldots, \gamma_{d+1, I}\right)$ for $\|I\| \leq d$. It is easy to see that $\mathcal{A}^{-1}=\left[\frac{1}{d+1} \varepsilon^{i j}\right]_{i, j=1, \ldots, d+1}$. So, for each $\left(\gamma_{1}, \ldots, \gamma_{n}\right) \in \mathbb{C}^{d+1}$, $\left|L^{-1}\left(\gamma_{1}, \ldots, \gamma_{d+1}\right)\right| \leq\left|\left(\gamma_{1}, \ldots, \gamma_{d+1}\right)\right|$. Hence and from the above we have the assertion.

5.8. Lemma. If $W$ is an m-dimensional irreducible algebraic subset of $\mathbb{C}^{m} \times \mathbb{C}^{\left(\begin{array}{c}d+n \\ n\end{array}\right)}$ such that the natural projection $\pi: W \ni(\lambda, y) \mapsto \lambda \in \mathbb{C}^{m}$ is proper, then the function

$$
(\lambda, x) \mapsto \prod_{(\lambda, y) \in \pi^{-1}(\lambda)} \mathcal{G}_{d}^{\text {mult }_{(\lambda, y)}(\pi)}(y, x), \quad \lambda \in \mathbb{C}^{m}, x \in \mathbb{C}^{n},
$$

is continuous.

Pro of. By the definition of multiplicity and the properties of $\pi$, we obtain $\sum_{(\lambda, y) \in \pi^{-1}(\lambda)} \operatorname{mult}_{(\lambda, y)}(\pi)=$ const. So, using once again the definition of multiplicity, we obtain the assertion.

Proof of Theorem 5.1. First, let us consider the case when $P$ is monic with respect to $X_{1}$ and take $G=1$. By Theorem 4.4 and Proposition 4.2 , we immediately see that there exist a positive integer $d$, an $m$ dimensional irreducible algebraic set $W_{1} \subset \mathbb{C}^{m} \times \mathbb{C}^{\left(\begin{array}{c}d+n \\ n\end{array}\right)}$, and a polynomial $Q \in \mathbb{C}[\Lambda]^{*}$, such that the projection $\pi_{1}: W_{1} \rightarrow \mathbb{C}^{m}$ is dominating and

$$
P(\lambda, X)=\prod_{(\lambda, y) \in \pi_{1}^{-1}(\lambda)} \mathcal{G}_{d}(y, X) \quad \text { for } \lambda \in \Omega_{Q}
$$

is a decomposition of $P(\lambda, X)$ into nonassociated irreducible factors in $\mathbb{C}[X]$ monic with respect to $X_{1}$, where $\mathcal{G}_{d}(y, X)$ has degree $d$. In particular, $\left.\pi_{1}\right|_{\pi_{1}^{-1}\left(\Omega_{Q}\right)}: \pi_{1}^{-1}\left(\Omega_{Q}\right) \rightarrow \Omega_{Q}$ is a covering, so $\operatorname{mult}_{(\lambda, y)}\left(\pi_{1}\right)=1$ for $(\lambda, y) \in$ 
$\pi_{1}^{-1}\left(\Omega_{Q}\right)$. Thus, we have (5.2) for $\lambda \in \Omega_{Q}$. Note that the projection $\pi_{1}$ is proper. Indeed, otherwise by the density of $\pi_{1}^{-1}\left(\Omega_{Q}\right)$ in $W_{1}$ (Prop. 4.2), there would exist a sequence $\left\{\left(\lambda_{n}, y_{n}\right)\right\} \subset \pi_{1}^{-1}\left(\Omega_{Q}\right)$ such that $\left|\left(\lambda_{n}, y_{n}\right)\right| \underset{n \rightarrow \infty}{\longrightarrow} \infty$ and $\left\{\lambda_{n}\right\} \subset \mathbb{C}^{m}$ is bounded. Then all the coefficients of $P\left(\lambda_{n}, X\right)$ are bounded and $\left|y_{n}\right| \underset{n \rightarrow \infty}{\longrightarrow} \infty$. This gives a contradiction with Lemma 5.5.

So, by the properness of $\pi_{1}$ and Lemma 5.8 , we conclude that the righthand side of (5.2) is a continuous function in $\mathbb{C}^{m} \times \mathbb{C}^{n}$. This, together with the density of $\Omega_{Q}$ in $\mathbb{C}^{m}$, gives (5.2) for any $\lambda \in \mathbb{C}^{m}$.

We now show the existence of an algebraic set $V$ satisfying the second part of the theorem. Let $l:=\operatorname{deg}_{X} P$. Take the algebraic set

$$
\begin{aligned}
A:=\left\{\left(\lambda, y^{1}, y^{2}\right) \in \mathbb{C}^{m} \times\right. & \mathbb{C}^{\left(\begin{array}{c}
l+n \\
n
\end{array}\right)} \times \mathbb{C}^{\left(\begin{array}{c}
l+n \\
n
\end{array}\right): P(\lambda, X)=\mathcal{G}_{l}\left(y^{1}, X\right) \mathcal{G}_{l}\left(y^{2}, X\right),} \\
& \mathcal{G}_{l}\left(y^{1}, X\right), \mathcal{G}_{l}\left(y^{2}, X\right) \text { are monic with respect to } X_{1} \\
& \text { and } \left.0<\operatorname{deg} \mathcal{G}_{l}\left(y^{1}, X\right)<d\right\} .
\end{aligned}
$$

Since the factors $\mathcal{G}_{d}(y, X)$ in (5.9) are irreducible for $\lambda \in \Omega_{Q}$, the projection of $A$ on $\mathbb{C}^{m}$ is contained in $\mathbb{C}^{m} \backslash \Omega_{Q}$. Hence by the Chevalley Theorem [E], Cor., p. 395, it is contained in a proper algebraic set $V \subset \mathbb{C}^{m}$. Consequently, for $\lambda \in \mathbb{C}^{m} \backslash V$ the right-hand side of (5.2) is a decomposition of $P(\lambda, X)$ into irreducible factors in $\mathbb{C}[X]$ monic with respect to $X_{1}$. Moreover, by the irreducibility of $P$, we may assume that, for $\lambda \in \mathbb{C}^{m} \backslash V$, the discriminant of $P$ with respect to $X_{1}$ is a nonzero polynomial. This implies that the factors on the right-hand side of (5.2) are not associated and $\operatorname{mult}_{(\lambda, y)}\left(\pi_{1}\right)=1$ for each $(\lambda, y) \in \pi_{1}^{-1}(\lambda), \lambda \in \mathbb{C}^{m} \backslash V$.

Summing up, we have the assertion in this case.

Changing variables if necessary, we obtain the assertion in the case when $P$ is monic with respect to one of the variables $X_{i}, i=1, \ldots, n$.

Let us consider the general case. It is easy to show that there exists a linear change of variables $L: \mathbb{C}^{n} \rightarrow \mathbb{C}^{n}$ such that, for the polynomial

$$
\widetilde{P}(\Lambda, X):=P(\Lambda, L(X)),
$$

we have $l=\operatorname{deg}_{X_{1}} \widetilde{P}=\operatorname{deg}_{X} \widetilde{P}$. Then the coefficient $G_{0} \in \mathbb{C}[\Lambda]$ of $X_{1}^{l}$ in $\widetilde{P}$ is nonzero. So, there exists a polynomial $R \in \mathbb{C}[\Lambda, X]$ such that

$$
G_{0}^{l-1}(\Lambda) \widetilde{P}(\Lambda, X)=R\left(\Lambda, G_{0}(\Lambda) X_{1}, X^{\prime}\right)
$$

where $X^{\prime}=\left(X_{2}, \ldots, X_{n}\right)$. Hence $R$ is monic with respect to $X_{1}$ and $\operatorname{deg}_{X_{1}} R$ $=\operatorname{deg}_{X} R$. By the assumption on Spec $P$, we get that Spec $R$ is dense in $\mathbb{C}^{m}$. By (5.10) and (5.11), $R$ is irreducible in $\mathbb{C}[\Lambda, X]$. Then, by the first part of the proof, there exist $d$, an $m$-dimensional irreducible algebraic subset $W_{1} \subset \mathbb{C}^{m} \times \mathbb{C}^{\left(\begin{array}{c}d+n \\ n\end{array}\right)}$ and an algebraic subset $V \varsubsetneqq \mathbb{C}^{m}$ such that the projection 
$\pi_{1}: W_{1} \ni(\lambda, y) \mapsto \lambda \in \mathbb{C}^{m}$ is proper and

$$
R(\lambda, X)=\prod_{(\lambda, y) \in \pi_{1}^{-1}(\lambda)} \mathcal{G}_{d}(y, X) \quad \text { for } \lambda \in \mathbb{C}^{m} \backslash V
$$

is a decomposition of $R(\lambda, X)$ into nonassociated irreducible factors in $\mathbb{C}[X]$. Without loss of generality we may assume that $V\left(G_{0}\right) \subset V$.

Let $F=\left(P_{I}:\|I\| \leq d\right): \mathbb{C}^{m} \times \mathbb{C}^{\left(\begin{array}{c}d+n \\ n\end{array}\right)} \rightarrow \mathbb{C}^{\left(\begin{array}{c}d+n \\ n\end{array}\right)}$ be the polynomial mapping defined by $P_{I}(\Lambda, Y):=G_{0}^{i_{1}}(\Lambda) Y_{I}$ where $I=\left(i_{1}, \ldots, i_{n}\right)$. By the Chevalley Theorem $W_{2}:=\left\{(\lambda, F(\lambda, y)) \in \mathbb{C}^{m} \times \mathbb{C}^{\left(\begin{array}{c}d+n \\ n\end{array}\right)}:(\lambda, y) \in W_{1}\right\}$ is an algebraically constructible set. By the properness of the projection $\pi_{1}$, we easily see that it is a closed set, and thus, algebraic. Moreover, the projection $\pi_{2}: W_{2} \ni(\lambda, y) \mapsto \lambda \in \mathbb{C}^{m}$ is proper and dominating, so $\operatorname{dim} W_{2}=m$. What is more, by (5.10)-(5.12), we find that

$$
G_{0}^{l-1}(\lambda) P(\lambda, L(X))=\prod_{(\lambda, y) \in \pi_{2}^{-1}(\lambda)} \mathcal{G}_{d}(y, X) \quad \text { for } \lambda \in \mathbb{C}^{m} \backslash V
$$

is a decomposition of the left-hand side into nonassociated irreducible factors in $\mathbb{C}[X]$.

Let $\mathcal{G}_{d}\left(Y, L^{-1}(X)\right)=\sum_{\|I\| \leq d} \widetilde{L}_{I}(Y) X^{I}$. It is easy to see that the map$\operatorname{ping} \tilde{L}:=\left(\tilde{L}_{I}:\|I\| \leq d\right): \mathbb{C}^{\left(\begin{array}{c}d+n \\ n\end{array}\right)} \rightarrow \mathbb{C}^{\left(\begin{array}{c}d+n \\ n\end{array}\right)}$ is linear and $\mathcal{G}_{d}\left(Y, L^{-1}(X)\right)=$ $\mathcal{G}_{d}(\tilde{L}(Y), X)$. Let us take the algebraic set $W=\left\{(\lambda, \widetilde{L}(y)) \in \mathbb{C}^{m} \times \mathbb{C}^{\left(\begin{array}{c}d+n \\ n\end{array}\right)}\right.$ : $\left.(\lambda, y) \in W_{2}\right\}$. Since $\pi_{2}$ is proper and dominating, so is $\pi: W \ni(\lambda, y) \mapsto \lambda \in$ $\mathbb{C}^{m}$, and hence $\operatorname{dim} W=m$. Thus, taking $G=G_{0}^{l-1}$, by (5.13), we obtain that

$$
G(\lambda) P(\lambda, X)=\prod_{(\lambda, y) \in \pi^{-1}(\lambda)} \mathcal{G}_{d}(y, X) \quad \text { for } \lambda \in \mathbb{C}^{m} \backslash V
$$

is a decomposition of the left-hand side into nonassociated irreducible factors in $\mathbb{C}[X]$. Hence, by Lemma 5.8, analogously as before we obtain (5.2).

This ends the proof of Theorem 5.1.

Acknowledgements. I would like to cordially thank Prof. Jacek Chạdzyński and Dr. hab. Tadeusz Krasiński for their valuable remarks and advice during the preparation of this paper.

\section{References}

[K] W. Krull, Über einen Irreduzibilitätssatz von Bertini, J. Reine Angew. Math. 177 (1937), 94-104.

[Ł] S. Łojasiewicz, Introduction to Complex Analytic Geometry, Birkhäuser, Basel, 1991. 
[M] D. Mumford, Algebraic Geometry I, Complex Projective Varieties, Springer, Berlin, 1976.

[N] R. Narasimhan, Several Complex Variables, Chicago Lectures in Mathematics, Chicago-London 1971.

[Sc] A. Schinzel, Selected Topics on Polynomials, The University of Michigan Press, Ann Arbor, 1982.

[Sp] S. Spodzieja, On multi-valued algebraic mappings, Bull. Soc. Sci. Lett. Łódź 44 (1994), 95-109.

[St] Y. Stein, The total reducibility order of a polynomial in two variables, Israel J. Math. 68 (1989), 109-122.

[T] P. Tworzewski, Intersection of analytic sets with linear subspaces, Ann. Scuola Norm. Sup. Pisa (4) 17 (1990), 227-271.

[W] R. J. Walker, Algebraic Curves, Dover, New York, 1950.

Faculty of Mathematics

University of Łódź

Banacha 22

90-238 Łódź, Poland

E-mail: spodziej@krysia.uni.lodz.pl 